\title{
A Study on Fast Preparation of Electron Microscope Biological Specimens
}

\author{
Guo Huashan, Yao Hongjun, Wu Yang, Yang Tian, Zhang Wei, and Guo Jianjun \\ College of Life Science, Huazhong Normal University, Wuhan 430079, China \\ guohuashan1982@sohu.com
}

\begin{abstract}
The electron microscope is widely used in chemistry, physics and biology fields, especially in biomedicine ultrastructure research. Using the ultrasonic waves, the time that we spend in preparing the biological specimen will be decreased from 5 10 days to 3 5 hours. A few investigation and discussion of fast preparation was proposed in the paper. This work is very significant to biomedicine research and clinic pathology diagnoses aspects. It is better to choose amplitude with low frequency for the purpose of fast preparation.
\end{abstract}

Keywords: Electron Microscope, Biology Samples, Preparation, Specimen.

\section{Introduction}

The electron microscope is widely used in chemistry, physics and biology fields, especially in biomedicine ultrastructure research. It is very important to know alternation of morphological structure to detail, but preparations of biology samples require cockamamie procedure before they can be observed by electron microscope. Routine preparation costs at least 5 days, even 10 days long or longer. In order to save time, reduce work, and improve working efficiency, the author has engaged in electron microscope technology for more than twenty years. A few investigation and discussion of fast preparation was proposed in the paper. This work is very important to biology science research and clinic pathology diagnoses aspects.

\section{Materials and Methods}

\subsection{Laboratory Apparatus and Reagents}

(1) Apparatus ultrasonic cleaner: An output of 50 watts, vibration frequency of 40 $\mathrm{KHz}$.

(2) Reagents. Paraform+glutaraldehyde mixed fixation, $0.2 \mathrm{~mol}$ PBS, $1 \%$ osmium tetroxide, 30\%, 50\%, 60\%, 70\%, 80\%, 90\%, 95\%, 100\% ethanol, acrylic propane, Epon812 acrylic resin.

To ensure that the reproduction of your illustrations is of a reasonable quality, we advise against the use of shading. The contrast should be as pronounced as possible. 
If screenshots are necessary, please make sure that you are happy with the print quality before you send the files.

\subsection{Preparation}

Before preparation, two-thirds volume of ice water is infused to flume of ultrasonic cleaner as driving medium. Glass vials containing specimens are placed into the flume. Here, taken the treatment of myocardium tissue of M.m.albula, kidney cortex, liver tissue, leaf tissue of photinia serrulata as an example.

(1) Pre-fixation

First, cutting material into $1 \mathrm{~mm} 3$ or smaller pieces, putting them in vial containing fixation solution as soon as possible, and then placing them into $4^{\circ} \mathrm{C}$ freezer.

Choosing fast-penetrating fixation solution, such as paraform+glutaraldehyde mixture, acrolein+glutaraldehyde mixture. After 20 minutes, putting vials containing specimens into flume of ultrasonic cleaner and start ultrasonic cleaner for 10 minutes.

(2) Washing

Discarding fixation solution, washing by $0.1 \mathrm{~mol}$ PBS to remove the fixation solution leaving on the surface of specimens, treating by ultrasonic for 4-6 minutes.

(3) Post-fixation

Post-fix specimens by $1 \%$ osmium tetroxide after washing, placing them into $4{ }^{\circ} \mathrm{C}$ freezer for 20 minutes, and then treating by ultrasonic for 10 minutes. This step should add ice to flume to keep the temperature down or cold-cycle water.

(4) Washing

Method is as same as (2).

(5) Dehydratation and staining

Dehydrating by ethanol in grads. Treating by $50 \%, 70 \%$ (containing $2 \%$ uranylacetate), $80 \%, 90 \%, 95 \%, 100 \%$ ethanol, each concentration for 3 minutes, and then treating by acrylic propane for 3 minutes.

(6) Permeance

The ratio of acrylic propane to Epon 812 acrylic resin is $1: 1$. Treating by ultrasonic for 10 minutes, and then treating by pure Epon 812 for 10 minutes. This step is important for embedding reagent to penetrate specimens fast.

(7) Embedding and polymerization

The specimens after permeance are better placed on warm table, put into capsules, and then embed in pure Epon812. Polymerizing specimens after placing them in 90$100^{\circ} \mathrm{C}$ incubator for 1 hour.

It can be concluded that the whole process costs less than 4 hours from fixation to polymerization. After polymerizing, embedded specimens can be used for trimming and sectioning, staining, observing and taking photos by electron microscope. 


\section{Results}

Compared ultrasonic prepared specimens with routine prepared specimens, according to the electron microscopic pictures, structure of kidney cortex and liver cell of M.m.albula, mitochondrion and myofibril in myocardium cell are preserved perfectly. Similarly, cell wall, plasmodesmatas of plant cells, especially grana and stroma of chloroplasts, have a normal configuration. In conclusion, this is an improved and efficient method to prepare electron microscope specimens of both plant and animal cell (Fig.1, Fig.2).

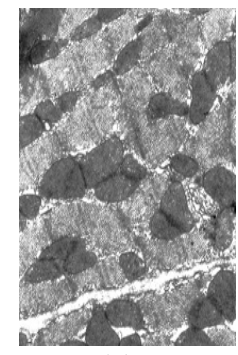

(a)

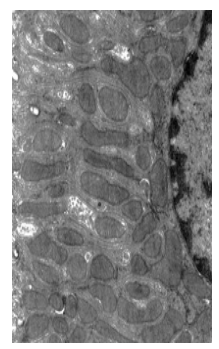

(b)

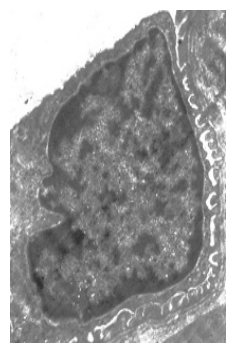

(c)

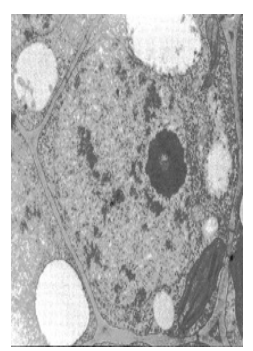

(d)

(a).Cardiac muscle of M.m albula( Bar=10 $\mu \mathrm{m}$ ) (b).liver of M.m albula( Bar=10 $\mu \mathrm{m}$ )

(c).Kidney cortex of M.m albula ( Bar=10 $\mu \mathrm{m}$ ) (d).leaf of photinia scrrulata ( Bar=10 $\mu \mathrm{m}$ )

Fig. 1. Ultrasonic prepared specimens

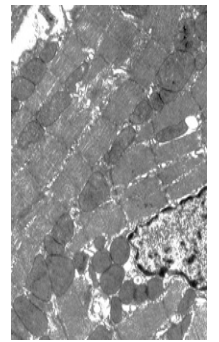

(a)

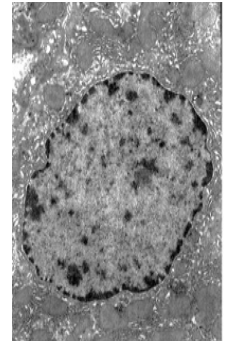

(b)

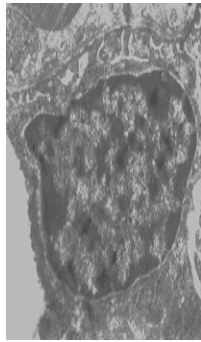

(c)

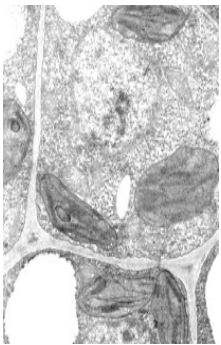

(d)

(a).Cardiac muscle of M.m albula ( Bar=10 $\mu \mathrm{m}$ ) (b).liver of M.m albula ( Bar=10 $\mu \mathrm{m}$ )

(c).Kidney cortex of M.m albula ( Bar=10 $\mu \mathrm{m}) \quad$ (d).leaf of photinia scrrulata ( Bar=10 $\mu \mathrm{m}$ )

Fig. 2. Ultrasonic prepared specimens

\section{Discussion and Conclusion}

There have been a few reports about fast preparation, Coulter made use of diminishing sample bulk, reducing dehydration time and vacuum imbuing method. However, some steps had shortened time [1]. Robbins and G.Jentzsch used Epon812 to steep monolayer cell, used 3 times solvent of embed, every time costs 5 minutes, the whole press costs 4 days.[3][4][5][6] The scholars who have engaged in fleetness 
preparation in homeland are also the same. The author is able to shorten preparation time, because ultrasonic is used to routine preparation procedure.

The author has done research on the applicability of this device, different processing method of different samples. Generally speaking it takes longer to process plant tissue than animal tissue, and it takes longer to process precise faction tissue than rare faction tissue. Meanwhile, the processing method is also suitable for scanning electron microscope specimen; however, it's difficult to process the primary embedded samples, because ultrasound can detach the primary embedded material from samples.

Ultrasonic is not only used for electron microscope biology sample preparation, but also may be used for organization slice up of the light microscope, some problems need further research. In hospital, frequency of the instrument for ultrasonic diagnosis and remedy in use is between 800 and $1000 \mathrm{KHz}$, while ultrasonic frequency used for electron microscope sample. Preparation is in $40 \mathrm{KHz}$. could it be raised, or what is the extremity? The best frequency and amplitude (in short, strength), and the exact length of time to process need further research. Because ultrasonic strength is pretty important to biology sample preparation, using different strength will cause different results. If the strength doesn't exceed the limit, longer time of processing will not cause the impairment of cells and tissue. Otherwise, if the strength exceed extremely, it will cause impairment of tissue. Moreover, selection of conductive transmitter and the decreasing quantity of its vibrational frequency after transmitter flowing into sample bottle need to research further. The author suggests on that on the basis of not damaging cell structure, it is better to choose amplitude with low frequency for the purpose of fast preparation.

\section{References}

1. Coulter M D. J.:U1trastruct Res. 1967, 346-347.

2. Robbins G, Jentzsch J.:Histochem.Cytochem. 1967, 181-182.

3. Zheng R.:Practicality cytology technology. Beijing Scientific Press, 1980, 188-189.

4. Chen Shi, Peng Xueming: Biomedicine electron microscope technology. Social science Press of China, 1997.

5. Arturo González-Robles: Ultrastructure of trophozoites using fast freeze-fixation followed by freeze-substition J. of Electron Microscopy, 2001, 423-427.

6. Fu Honglan: Practical Electron Microscopy Beijing.Higher Education Press, 2004, 33-34. 